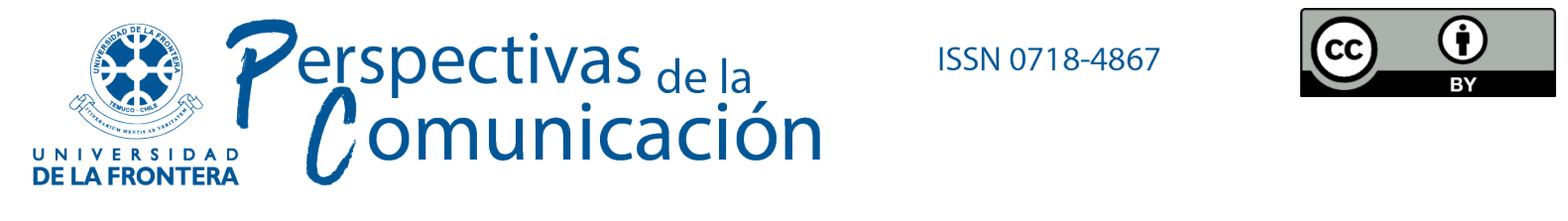

Artículo

\title{
EL DEBATE SOBRE LA LEGALIZACIÓN DEL ABORTO EN \\ LA CAMPAÑA ELECTORAL ARGENTINA DE 2019. UN \\ ANÁLISIS DESDE LA COBERTURA DE LOS PRINCIPALES MEDIOS DIGITALES DEL PAÍS.
}

DOI: POR ASIGNAR

\author{
Dra. Laura Rosenberg \\ Consejo Nacional de Investigaciones Científicas y Técnicas (CONICET) y Universidad Nacional de Avellaneda, \\ Buenos Aires, Argentina \\ laura.rosenberg85@gmail.com \\ ORCID iD: https://orcid.org/0000-0002-3002-5140
}

Recibido el 2020-12-18

Revisado el 2021-05-02

Aceptado el 2021-05-10

Publicado el 2021-07-12

\section{Resumen}

En 2018 ocurrió un hecho sorpresivo en Argentina. Por primera vez un presidente -de la derecha conservadora- habilitaba el debate sobre la legalización del aborto en el Congreso. Tras meses de debate en el parlamento, con la presencia de más de 700 expositores que manifestaron posturas a favor y en contra de la iniciativa, el proyecto fue rechazado por escaso margen en el Senado. Pero el tema se había instalado en la agenda pública, y se esperaba que haga lo propio en la agenda política del año electoral entrante.

Desde los enfoques de agenda setting y framing, se llevó a cabo un análisis de contenido de corte cuantitativo para indagar en la relevancia y el tratamiento que tuvo el debate por la legalización del aborto en las coberturas informativas sobre las elecciones de los medios digitales más consultados a nivel nacional (Clarín, Infobae y La Nación). 
Perspectivas de la Comunicación

Universidad de La Frontera

Palabras clave: aborto, agenda mediática, elecciones, agenda setting, framing, análisis de contenido. 


\title{
THE DEBATE ON THE LEGALIZATION OF ABORTION IN THE ARGENTINIAN ELECTORAL CAMPAIGN IN 2019. AN ANALYSIS ON THE COVERAGE OF THE MAIN DIGITAL MEDIA IN THE COUNTRY.
}

\begin{abstract}
A surprising event occurred in Argentina in 2018. For the first time, a president -of the conservative right- enabled in Congress the debate on the legalization of abortion. After months of debate, with the presence of more than 700 speakers who expressed positions in favor and against the initiative, the project was rejected by the Senate. But the issue had been installed on the public agenda, and it was expected to do the same on the political agenda for the election year next year.

From the agenda setting and framing approaches, a quantitative content analysis was carried out to research the relevance and treatment of the debate on the legalization of abortion in the news coverage on the elections of the most consulted digital media nationwide (Clarín, Infobae and La Nación).
\end{abstract}

Keywords: abortion, media agenda, elections, agenda setting, framing, content analysis. 


\section{Introducción}

El 9 de agosto de 2018, luego una extensa sesión, el Senado argentino rechazó el proyecto de Ley de Interrupción Legal del Embarazo (ILE) al cual la Cámara de Diputados había otorgado media sanción dos meses atrás. La votación definió el resultado de una serie de acontecimientos y disputas entre actores sociales, políticos y religiosos que impregnaron la agenda pública desde el mes de marzo de aquel año en Argentina. Por aquel entonces, en el marco de la inauguración de las sesiones parlamentarias, el presidente Mauricio Macri planteó que estaba "a favor de la vida, pero también de los debates maduros y responsables" sobre el aborto en el país, promoviendo de este modo su inclusión en la agenda legislativa.

Aquel anuncio resultó sorpresivo para propios y ajenos a la coalición gobernante. Más allá de los motivos políticos y pragmáticos subyacentes (Vommaro, 2018; Alconada-Mon, 2018), lo cierto era que un presidente de la derecha conservadora y neoliberal (Svampa, 2019) -que seis años atrás y como jefe de gobierno en la Ciudad de Buenos Aires había vetado una ley de aborto no punible- habilitaba el avance sobre una de las cuestiones más relevantes para el movimiento feminista. Disputaba así un terreno sobre el cual su antecesora, Cristina Fernández de Kirchner, se había negado a incursionar (Alcaraz, 2019) y que a ella le había valido no pocas críticas desde los sectores progresistas del peronismo y de otros partidos de la izquierda en Argentina.

A pesar de los resultados de la votación en el Congreso, los hechos suscitados tanto dentro del parlamento como puertas afuera, en las masivas movilizaciones en las calles, significaron un hito para el movimiento feminista en el país (Alcaraz, 2019; Peker, 2019; Tarducci, 2018). Nunca un proyecto sobre legalización del aborto había llegado tan lejos en el Congreso ni se había instalado simultáneamente y con tal relevancia en las agendas pública, política y mediática, dando lugar a lo que el movimiento entendió como una "despenalización social del aborto" (Tarducci, 2018, p. 430).

Activistas, especialistas e investigadoras sobre el acceso de las mujeres a los derechos sexuales y reproductivos advirtieron que el debate había calado hondo en la sociedad y que la derrota en el Senado no representaba un punto final. Era más bien una instancia significativa de la larga lucha que las mujeres habían comenzado varias décadas atrás, y que en el año 2007 había comenzado a tomar forma de proyecto de Ley cuando la Campaña Nacional por el Derecho al Aborto presentó su primer proyecto en el parlamento (Tarducci, 2018). Quienes se habían pronunciado a favor de la iniciativa visualizaban que lo ocurrido 
el 9 de agosto de 2018 no representaba en modo alguno una derrota. Por el contrario, auguraba la legalización de la interrupción voluntaria del embarazo en un futuro cercano.

Por caso, minutos antes de la votación, el senador Pino Solanas se dirigía en su discurso en el recinto a las manifestantes a favor de la legalización que aguardaban afuera del Congreso: "Hoy no es una derrota. Se los digo a las chicas que están afuera. Este es un triunfo monumental, porque hemos logrado instalar[lo] en el debate nacional... iellas han logrado!”. La tapa del diario Página $/ 1^{1}$ mostraba en edición del 9 de agosto una imagen de manifestantes a favor de la legalización junto al título "Nadie puede para el viento", haciendo alusión al sentimiento de esperanza que dejaba el resultado negativo de la votación dentro y fuera del Senado. Contrarrestando la derrota puertas adentro del parlamento, la periodista Mariana Carbajal (2018) escribía en su columna: "Ganamos”. El debate había dejado su impacto:

Más allá del resultado adverso del tratamiento de la ley, lo más significativo que se ha vivido en la sociedad argentina ha sido la movilización excepcional de millones de manifestantes, sobre todo, de adolescentes y de jóvenes, y la adhesión multitudinaria al símbolo del pañuelo verde que sintetiza la convicción de que la legalización del aborto constituye un derecho humano fundamental. Se ganó en la calle lo que se perdió en el recinto senatorial, y ya nada será como antes (Barrancos, 2018, p. 375).

El debate en sí mismo generó una grieta cultural (Vommaro, 2018) y transversal (Amadeo, 2018, p. 107) donde la intervención de actores políticos, culturales y religiosos cruzó ambos lados de la tradicional grieta que los últimos años caracterizaba la política argentina, entre kirchneristas y antikirchneristas: "El tema del aborto dio lugar a otros conglomerados políticos. El origen partidario dejó de ser un indicador de posicionamiento frente a este tema" (Amadeo, 2018, p. 108).

\footnotetext{
${ }_{1}$ Página/12 es un diario argentino de alcance nacional que se publica desde 1987, cuando el país atravesaba la etapa de transición democrática luego de la última dictadura cívico militar (1976-1983). Desde entonces, se caracteriza por ser un medio orientado a un público de centro-izquierda con una agenda que hizo vanguardia en lo que respecta a la cobertura sobre derechos humanos.
} 
Los efectos del debate hicieron presuponer que la cuestión de género en términos generales y del aborto en particular tendrían un lugar relevante entre los tópicos de la agenda política y mediática en el contexto electoral del año entrante. Sería algo inédito: los candidatos no podrían soslayar la expresión de su postura sobre el asunto. Pero lo cierto es que la permanencia de temas controversiales en esas agendas puede dar lugar a jugadas riesgosas en momentos críticos para el arco político, donde a lo que se aspira es a la conformación de alianzas electorales entre distintas fuerzas, que no tenían precisamente un punto de encuentro en esta materia.

¿Cómo determinar el impacto que podía generar en el amperímetro electoral retomar el debate por la legalización del aborto? ¿No podría ello profundizar rivalidades entre actores del propio espacio y aproximar entre sí a candidatos de distintas fuerzas? Como se subrayó anteriormente, la transversalidad política de estas cuestiones podía motivar divisiones internas que ningún candidato con chances reales de acceder al poder estaba dispuesto a afrontar. En efecto, para evitar este escenario, los partidos mayoritarios equilibraron la composición de sus listas alternando en ellas a "verdes" y “celestes"2.

Distintos análisis caracterizaron el escenario de las elecciones de 2019 a partir de la profunda polarización entre las dos primeras fuerzas políticas -la oficialista Juntos por el Cambio y la opositora Frente de Todos que juntas cosecharon más del 80\% de los votos- y de la agudización de la crisis económica (Garriga \& Negri, 2020; Koziner, 2020; Ratto, 2020; Reina, 2019; Svampa, 2019) que puso en jaque la credibilidad del gobierno macrista y socavó sus chances de ganar las elecciones (Falcone, 2019).

A pesar de los intentos del oficialismo por desviar la centralidad de la cuestión, la crisis económica se impuso entre las preocupaciones de los votantes y en las críticas del arco

\footnotetext{
${ }^{2}$ La utilización de pañuelos verdes o celestes se erigió en 2018 como símbolo de las posturas enfrentadas en torno a la legalización del aborto. El pañuelo verde es el emblema de la Campaña Nacional por el derecho al aborto desde hace varios años; su estampa contiene el lema "Educación sexual para decidir. Anticonceptivos para no abortar. Aborto legal para no morir”. El pañuelo celeste, por el contrario, representó a los sectores que se opusieron a la legalización; autodenominados "provida”. En 2018 era habitual ver en las calles mujeres llevando uno de estos dos pañuelos, expresando posicionamiento acerca del debate que se daba en el Congreso.
} 
opositor. Lo económico se convirtió en el tópico más relevante (Koziner, 2020) y en factor explicativo de la derrota del oficialismo en las urnas (Garriga \& Negri, 2020; Ratto, 2020).

Tal como apunta Koziner (2020, p. 50), entre abril y octubre de 2019, los tópicos más relevantes en la agenda de los principales medios digitales -Clarín, Infobae y La Naciónfueron las elecciones (27,7 \% de las noticias), la economía (22,6 \% de las noticias) y la inseguridad (11,6 \% de las noticias). Muy por detrás se encontraron las noticias relacionadas a la cuestión de género, que representaron solo el $2 \%$ del total. En vistas a estos datos, a continuación, formulamos los interrogantes que guiaron nuestra indagación sobre la inclusión del aborto en las agendas pública, política y mediática en la coyuntura electoral argentina.

\subsection{Unidades de contexto, preguntas y objetivos de investigación}

En este artículo nos proponemos analizar la relevancia y el tratamiento que recibió la legalización del aborto como tópico de discusión en el marco de la campaña electoral de 2019, a partir de la cobertura de los principales medios periodísticos digitales: clarin.com, infobae.com y lanacion.com. Estos medios conforman nuestras unidades de contexto.

Clarín y La Nación tienen su origen en el papel y es en el terreno de la prensa gráfica donde consolidaron su lugar dominante en el mercado periodístico en el siglo XX, posición que mantienen en el siglo XXI a pesar de la crisis de los medios gráficos y gracias a la expansión que tuvieron sus respectivos conglomerados mediáticos en el sector de la infocomunicación, pero también en otras áreas económicas claves ${ }^{3}$. Estos medios son los de mayor tirada nacional, según los datos del Instituto Verificador de Circulaciones (IVC) (2020). En términos de estructura económica, Clarín se distingue por el ser multimedio más importante del país (dueños de medios impresos, digitales, radios, canales de televisión, cableoperadores y servicios de telefonía) lugar que conserva gracias al inédito proceso de expansión que alcanzó desde fines del siglo XX hasta la actualidad (Becerra \& Mastrini, 2017; MEDIA OWNERSHIP MONITOR (MOM), 20194).

3 En efecto, ambos grupos son socios en Expo Agro, la exposición rural más importante de Argentina (Zunino, 2016).

4 Como consigna en su informe Media Ownership Monitor Argentina (2019, p. 8): "El Grupo Clarín es el mayor de los grupos mediáticos, con presencia dominante en todas las industrias de medios y auxiliares (como es el caso de la fabricación y

Perspectivas de la Comunicación - Vol. 14 - No 1 - 2021 - pp. 113-139

Universidad de la Frontera - Chile 
A pesar del lugar consolidado que Clarín y La Nación detentan en el mercado de medios, en el terreno digital Infobae les disputa hegemonía. Nació como medio digital en 2002 y rápidamente se instaló entre los medios periodísticos más consultados en Internet junto a clarín.com y lanación.com (Alexa, 2020; MOM, 2019). A este factor se agregan otras características compartidas entre estos medios digitales: su tendencia generalista y sus líneas editoriales políticamente afines a la centroderecha. Se considera que estos aspectos tornan comparables los casos y dotan de relevancia a las preguntas de investigación que guiaron este trabajo, a saber:

a) ¿Qué lugar ocupó el tópico legalización del aborto en la cobertura mediática de la campaña presidencial de 2019?

b) ¿Se condice la visibilidad de este tópico durante la campaña con la repercusión social y política que tuvo un año atrás, en 2018?

c) ¿Cuáles son las características del tratamiento del aborto como tópico durante la campaña presidencial argentina por parte de los principales medios digitales del país?

De este modo, formulamos como objetivo general analizar la visibilidad del tópico legalización del aborto en la cobertura realizada por los principales medios digitales del país sobre la campaña presidencial argentina del año 2019. Específicamente, se indagará sobre: la presencia del tópico entre las noticias más relevantes de cada medio; los actores involucrados en estas noticias, los escenarios donde manifestaron sus posturas sobre el tema y la valorización positiva, negativa o indefinida que los actores realizan en el marco de la campaña electoral acerca del tópico legalización del aborto.

\subsection{Marco teórico}

Para cumplir nuestros objetivos, este artículo se basa en el enfoque de las teorías de la agenda setting y del framing, específicamente en lo relativo al estudio de la relevancia de

comercialización de papel, dado su control de Papel Prensa SA, sociedad que comparte con La Nación y con el Estado Nacional). Además, en los dos últimos años amplió su área de incumbencia al protagonizar la mayor fusión de la historia de las comunicaciones en el país, a través de la sociedad Cablevisión - Telecom avalada por el presidente Mauricio Macri. Con ello, el Grupo Clarín obtuvo un poder significativo de mercado también en el campo de las telecomunicaciones y de la conectividad (fija y móvil) a Internet”. 
los temas en las agendas mediáticas y algunas de sus propuestas para abordar el tratamiento que le dan los medios. Ambas perspectivas destacan el rol que juegan los medios de comunicación en el debate público, a la vez que se distancian tanto de enfoques previos que describían esa relación en términos de total determinación como de aquellos que desestimaban la influencia que los medios pueden tener en la opinión pública5.

En relación con el primero de los enfoques, el trabajo se sitúa en la primera fase de análisis de la teoría de la agenda setting. Para comprender esta instancia cabe recordar la repercusión que tuvo el trabajo pionero de esta escuela, de McCombs y Shaw (1972), quienes pusieron a prueba la hipótesis de Bernard Cohen que sostenía que los medios no determinan lo que pensamos, pero sí influyen sobre qué asuntos hacerlo. Los autores demostraron que las cuestiones predominantes en los medios de comunicación se tornaban relevantes para la población. Como destacó Aruguete (2015) en esta fase de análisis la perspectiva demuestra empíricamente el efecto cognitivo de los medios de comunicación, reafirmando que: "los medios tienen la capacidad de transferir la importancia otorgada a determinados objetos desde su agenda hacia la opinión pública” (p. 58).

Si bien nuestro artículo no se centrará en la dimensión de los efectos del mensaje mediático, el aporte de los estudios de agenda setting abre el interrogante sobre las consecuencias prácticas que pueden derivarse del hecho de que los medios iluminen ciertos temas por sobre otros. Esto, en definitiva, da cuenta de la articulación que existe entre la agenda mediática con la política y la pública (Aruguete, 2015), y explica por qué es importante estudiar lo que ocurre en la primera para entender la visibilidad que algunos temas adquieren en ciertas coyunturas político-sociales.

Para el caso que nos convoca: ¿qué consecuencias puede acarrear que el aborto aparezca como un tema relevante en la campaña presidencial? ¿puede ello repercutir en el interés que le asigne la opinión pública al posicionamiento de los candidatos sobre el tema y, así, en la

${ }^{5} \mathrm{Al}$ respecto, resultaban representativas del primer abordaje la "teoría de la aguja hipodérmica" de Harold Laswell, y la "ley de las mínimas consecuencias” enunciada por Paul Lazarfeld, del segundo abordaje. Aruguete (2015) brinda una descripción detallada de los contrapuntos de la teoría de la agenda setting con estos otros enfoques de la primera mitad del siglo XX sobre el rol de los medios de comunicación. 
consideración sobre a quién dirigir el voto? En cambio, ¿qué puede significar que el tema no tenga en la campaña presidencial la relevancia que se esperaba que tuviera un año atrás? Atendiendo entonces al aporte de la primera fase de análisis de la teoría de la agenda setting, en este artículo se medirá la relevancia que tuvo el tópico aborto en la cobertura de los tres principales medios digitales sobre la campaña presidencial.

Atento a la escasez de estudios desde el enfoque agenda setting en nuestro país, Zunino (2016) sistematizó los indicadores para medir la importancia de un asunto en las coberturas periodísticas -entre los cuales menciona la frecuencia y la jerarquía informativa. En articulación con la propuesta de los antecedentes en materia de agenda setting, el autor retoma de Amadeo (2018) el concepto de relevancia, al cual entienden por la: "visibilidad de la información a partir de su ubicación, su tamaño, su disposición con respecto a otro tipo de información o su mayor frecuencia de cobertura" (Amadeo, 2008; citado en Zunino, 2016, p. 129). Se distinguen aquí dos factores a considerar para el análisis de la relevancia de un tema o tópico en la cobertura noticiosa. El primero remite a la frecuencia, que permite identificar la cantidad de notas publicadas sobre un tema; el segundo da cuenta de la jerarquización, a partir de la ubicación de dichas notas en la publicación, considerando su extensión y los recursos paratextuales que se incorporan. Estos aspectos serán los considerados en este trabajo al momento de analizar la relevancia del tópico aborto en la cobertura informativa sobre las campañas presidenciales de 2019 en Argentina, sobre lo cual ampliaremos en el apartado metodológico.

En una segunda instancia, como se ha planteado anteriormente, este trabajo indaga sobre el tratamiento que recibió el tema en cuestión a partir de un análisis cuantitativo del contenido de las noticias que tienen por tópico principal o secundario el aborto. Para ello, recuperamos la propuesta de la teoría del framing en medios de comunicación o encuadre de las noticias que, desde un enfoque constructivista, se abocó a comprender "los significados de la realidad difundidos desde los medios de comunicación y su repercusión en los significados de esa misma realidad para las audiencias" (Sádaba-Garraza, 2001, p. 156). Ello implicará realizar un análisis de contenido sobre los textos de las noticias, cuyas especificidades se desarrollarán en el apartado metodológico.

Conceptualmente se entenderá el encuadre como una práctica que "implica seleccionar y realzar algunos aspectos de eventos o temas, y hacer conexiones entre ellos para promover una interpretación, evaluación y/o solución. Las palabras e imágenes que componen un 
encuadre pueden ser distinguidas de las demás noticias por su capacidad para estimular apoyo u oposición a los distintos campos de un conflicto político" (Entman, 2003; citado en Koziner \& Aruguete, 2020, p. 209). En este trabajo focalizaremos la atención en la arena desde la cual se hace referencia a la cuestión del aborto, los actores involucrados y el tono valorativo sobre el tópico que predomina en las noticias.

\section{Método}

Para cumplir el objetivo de analizar la relevancia y el tratamiento de la legalización del aborto como tópico en las coberturas informativas sobre la campaña electoral del año 2019, se implementará un estudio de corte cuantitativo atento a las pautas de trabajo de las teorías de la agenda setting y del framing.

En relación con las técnicas de recolección de datos, se recurrió como punto de partida al relevamiento realizado en dos etapas por los Observatorios de Medios de la Universidad Nacional de Cuyo, de la Universidad Nacional de Quilmes y de la Universidad Nacional del Centro de la Provincia de Buenos Aires sobre las primeras 10 notas referidas a las elecciones y que fueron publicadas en los portales de los medios periodísticos digitales más consultados: infobae.com, clarin.com y lanancion.com.

La primera etapa del relevamiento se llevó a cabo entre el 14/7/2019 y el 18/8/2019, comprendiendo la fecha en la cual tuvieron lugar las elecciones primarias, abiertas y obligatorias (PASO), el día 11/o8/2019. La segunda etapa de recolección se llevó a cabo entre el 29/9/2019 y el 03/11/2019, comprendiendo la fecha en la cual tuvieron lugar las elecciones generales, el día 27/10/2019. El corpus resultante estuvo conformado por aquellas notas que relacionadas con las elecciones nacionales, provinciales y municipales y se encontraran entre las primeras diez notas de los sitios web de medios mencionados. Es decir que el corpus incluyó desde el primer momento aquellas las notas que cumplían las condiciones de jerarquización de las noticias.

Entre ambas etapas se reunió un corpus de 2084 piezas informativas de los medios en cuestión. De este universo, para el presente trabajo se seleccionaron aquellas notas donde se identificaron alusiones al tópico y/o actores directamente relacionados con el debate por la legalización del aborto. Como veremos más adelante, esta selección permitió recortar el corpus de manera considerable, por lo cual resultó factible hacer luego un análisis de contenido sobre el total de las piezas que abordaron el tópico. Como formula Koziner (2013),

Perspectivas de la Comunicación - Vol. 14 - No 1 - 2021 - pp. 113-139

Universidad de la Frontera - Chile 
al tratarse de un corpus más pequeño es posible realizar un análisis de carácter inductivo, cuyas dimensiones emergieron de los rasgos específicos de los textos sobre la problemática de estudio.

El análisis se dividió entonces en dos etapas. En la primera, se midió la frecuencia de aparición del tópico en la agenda informativa de los medios en los períodos señalados. Teniendo en cuenta que el corpus estaba conformado por notas cuya ubicación daba cuenta de su jerarquización en la agenda, sumado a la medición de la frecuencia se pudo analizar la relevancia del tópico en estos medios, acorde a la primera fase de análisis de la teoría de la agenda setting.

La segunda etapa del trabajo adoptó la perspectiva del framing para desarrollar un análisis de contenido de carácter inductivo de los textos periodísticos ${ }^{6}$ y evaluar así el tratamiento del tópico aborto en las noticias publicadas. El análisis se efectuó a partir de la identificación de los actores involucrados en las noticias, los escenarios donde expresan sus posturas relacionadas a la legalización del aborto, y la articulación entre dichas posturas con las propuestas de los partidos en relación con la problemática en el marco de la campaña electoral. Estas cuestiones se definieron como ejes analíticos que emergieron del corpus.

\section{Resultados}

Para explicar qué lugar ocupó el tópico aborto en la cobertura informativa de la campaña presidencial de 2019, se calcularon las frecuencias absoluta y relativa del tópico en las coberturas de los tres medios:

\footnotetext{
${ }^{6}$ Diversos autores han destacado el carácter integral de la perspectiva del framing, dadas las posibilidades que brinda de analizar el tratamiento noticioso desde la instancia de producción del mensaje hasta su recepción (Amadeo, 2008; Aruguete, 2015; Koziner, 2013). En este artículo se estudiará el frame específicamente a partir del análisis de los textos de las noticias.
} 
Tabla 1. Frecuencia absoluta y relativa del tópico aborto en las coberturas informativas de los medios clarin.com, infobae.com y lanacion.com en el período de las elecciones primarias

\begin{tabular}{l|l|l|l|lr} 
Medio & $\begin{array}{l}\text { Aborto } \\
\text { como tópico } \\
\text { principal }\end{array}$ & $\begin{array}{l}\text { Aborto como } \\
\text { tópico } \\
\text { secundario }\end{array}$ & $\begin{array}{l}\text { Total de } \\
\text { noticias } \\
\text { relevadas }\end{array}$ & $\begin{array}{l}\text { Presencia del } \\
\text { tópico sobre el } \\
\text { total de coberturas }\end{array}$ \\
\hline clarin.com & 0 & 4 & 344 & $1,2 \%$ \\
lanacion.com & 0 & 3 & 374 & $0,8 \%$ \\
infobae.com & 0 & 2 & 313 & $0,6 \%$ \\
Total & 0 & 9 & 1031 &
\end{tabular}

Fuente: elaboración propia en base a relevamientos realizados por los Observatorios de Medios de la Universidad Nacional de Quilmes, la Universidad Nacional de Cuyo y la Universidad Nacional del Centro de la Provincia de Buenos Aires.

Tabla 2. Frecuencia absoluta y relativa del tópico aborto en las coberturas informativas de los medios clarin.com, lanacion.com e infobae.com en el período de las elecciones generales

\begin{tabular}{l|l|l|l|lr} 
Medio & $\begin{array}{l}\text { Aborto } \\
\text { como tópico } \\
\text { principal }\end{array}$ & $\begin{array}{l}\text { Aborto como } \\
\text { tópico } \\
\text { secundario }\end{array}$ & $\begin{array}{l}\text { Total de } \\
\text { noticias } \\
\text { relevadas }\end{array}$ & $\begin{array}{l}\text { Presencia dér } \\
\text { tópico sobre el } \\
\text { total de coberturas }\end{array}$ \\
\hline clarin.com & 3 & 1 & 361 & $1,1 \%$ \\
lanacion.com & 1 & 0 & 396 & $0,3 \%$ \\
infobae.com & 2 & 4 & 296 & $2 \%$ \\
Total & 5 & 6 & 1053 &
\end{tabular}

Fuente: elaboración propia en base a relevamientos realizados por los Observatorios de Medios de la Universidad Nacional de Quilmes, la Universidad Nacional de Cuyo y la Universidad Nacional del Centro de la Provincia de Buenos Aires.

Como se desprende de las tablas 1 y 2, solo una escasa proporción de las noticias que han sido relevadas entre las primeras diez de la web de estos medios abordaron el tópico, con leves matices entre las coberturas de las elecciones PASO y las generales. Si se observan las frecuencias relativas en ambos períodos las mismas rondan el $1 \%$, con lo cual estamos en condiciones de afirmar que el tópico tuvo una presencia muy inferior a la esperada en 2018 en las coberturas sobre la campaña electoral de 2019, según surge del análisis de más de 2000 piezas informativas. 
Pero cabe destacar que, aun cuando la participación del tópico en la agenda no mostró variaciones significativas entre ambas etapas del relevamiento y análisis de las piezas informativas -coincidente con los períodos próximos a cada uno de los comicios-, y que no hubo un incremento significativo en su frecuencia, se advierte la aparición del asunto como tópico principal en las elecciones generales.

Como planteamos anteriormente, la existencia de un corpus acotado de noticias sobre el aborto posibilita un análisis sobre la totalidad de las piezas informativas para comprender el tratamiento que recibió la cuestión en las coberturas informativas sobre la campaña electoral. Para analizar el encuadre nos centramos en cuatro aspectos de la noticia: el escenario donde tuvo lugar la referencia al tópico; los actores que aparecen relacionados al tópico aborto y la cobertura sobre la valorización que estos actores hicieron sobre la cuestión en el marco de la campaña electoral. Es importante volver a destacar que se trata de piezas que han sido seleccionadas por su relevancia y relación con procesos electorales a nivel nacional, local y municipal de 2019 en Argentina.

\subsection{Los escenarios}

Gráfico 1: Frecuencia del ámbito desde el cual se alude al tópico aborto

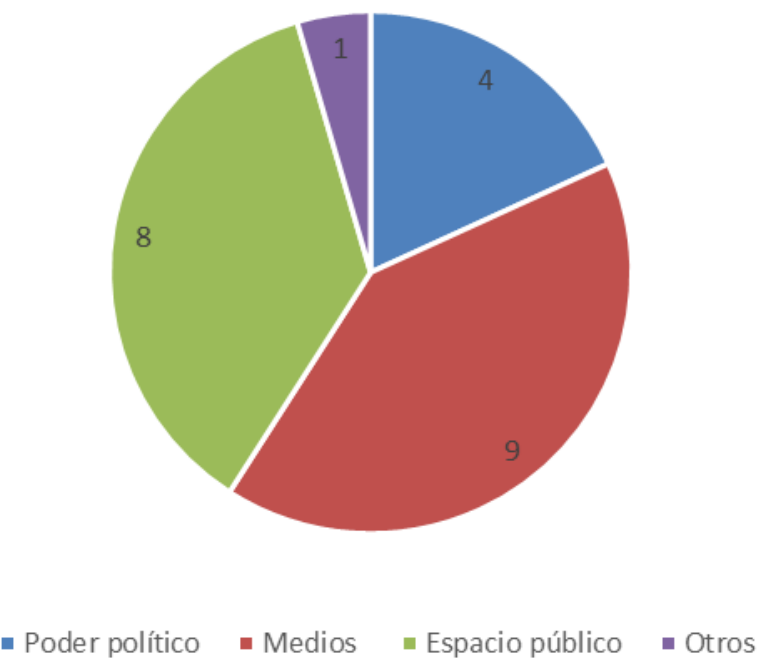

Fuente: Elaboración propia en base a relevamientos realizados por los Observatorios de Medios de la Universidad Nacional de Quilmes, la Universidad Nacional de Cuyo y la Universidad Nacional del Centro de la Provincia de Buenos Aires. 
Los medios y el espacio público resultaron ser las escenas donde más transcurrieron los acontecimientos narrados en las coberturas sobre la campaña electoral de 2019 de acuerdo con el tópico aborto. Con relación a la primera de las categorías, se trató de coberturas en las cuales los candidatos expresaron su postura sobre el aborto a los medios de comunicación y la presencia del tópico en el marco del primer debate presidencial transmitido por televisión. Respecto al espacio público, centralmente se refirió a actividades proselitistas donde candidatos de Juntos por el Cambio se mostraron con pañuelos celestes expresándose "a favor de las dos vidas", dando a entender su oposición a la legalización del aborto. En efecto, 6 de las 8 piezas informativas remiten a la primera vez que Mauricio Macri expresó en la campaña su rechazo a la legalización del aborto en un acto proselitista en la provincia de Mendoza. Más adelante cuando analicemos los datos de los gráficos 3 y 4 ampliaremos esta cuestión.

\subsection{Los actores}

En los textos de las noticias se hizo alusión al rol que jugaron diferentes actores en acontecimientos ligados a la campaña electoral con referencias al tópico del aborto. Como se observa en el siguiente gráfico, se destaca la presencia del presidente y candidato Mauricio Macri y del oficialismo nacional.

Gráfico 2: Frecuencia de cobertura de los actores en relación con el tópico aborto

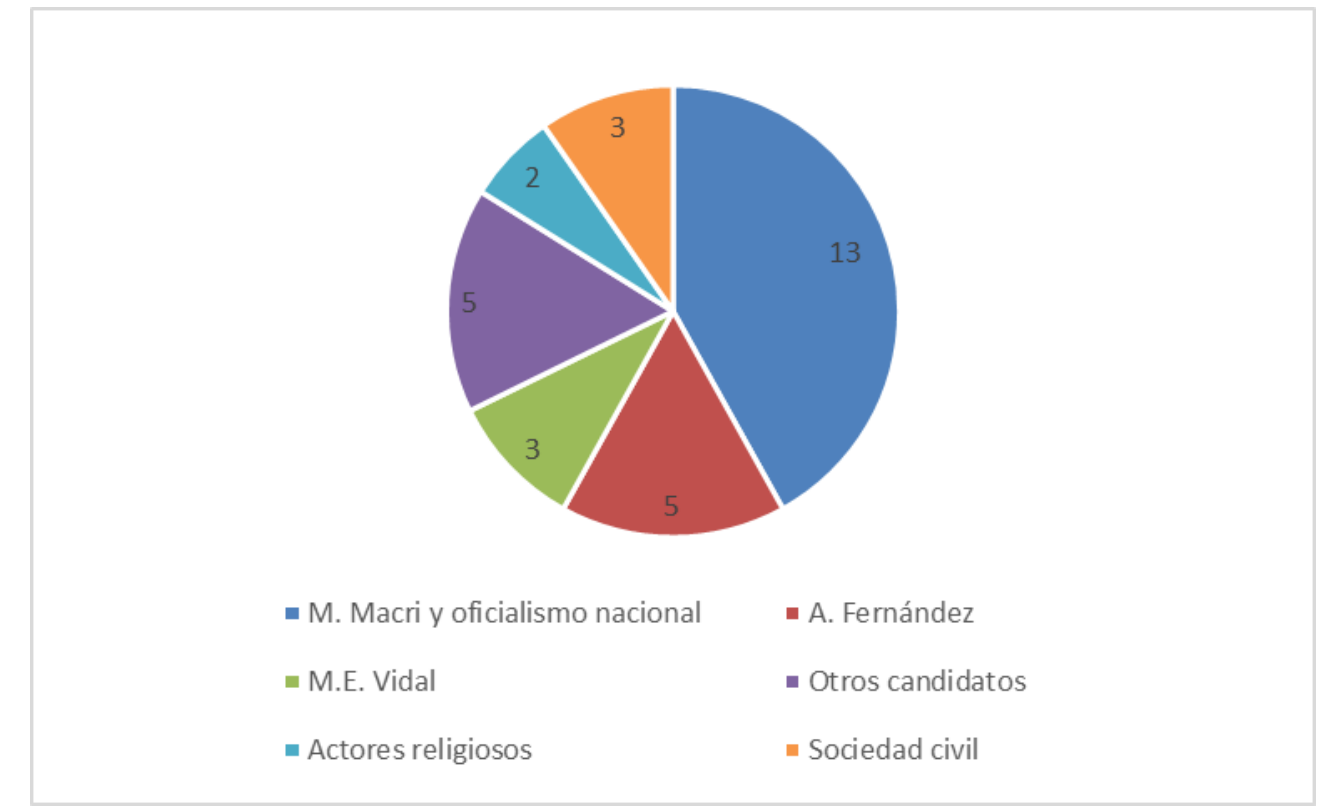

Perspectivas de la Comunicación - Vol. 14 - No 1 - 2021 - pp. 113-139

Universidad de la Frontera - Chile 
Fuente: elaboración propia en base a relevamientos realizados por los Observatorios de Medios de la Universidad Nacional de Quilmes, la Universidad Nacional de Cuyo y la Universidad Nacional del Centro de la Provincia de Buenos Aires.

Secundando las apariciones de Mauricio Macri y sus equipos de gobierno y de campaña se encuentran María Eugenia Vidal -gobernadora de la provincia de Buenos Aires y candidata al mismo cargo- y el candidato a presidente Alberto Fernández. Por último, se hace referencia a actores de la sociedad civil, a otros candidatos nacionales y municipales y a actores religiosos.

\subsection{La valorización de los actores sobre el tópico}

La dimensión valorativa resultó decisiva para comprender la disparidad entre la presencia de actores del oficialismo y de la oposición en las coberturas informativas observada en el gráfico 2, y para analizar el lugar que el tópico aborto ocupó en dichas coberturas en el marco de la campaña electoral de 2019.

Gráfico 3: Frecuencia del tópico aborto según la valorización que realizan los actores referidos en la noticia.

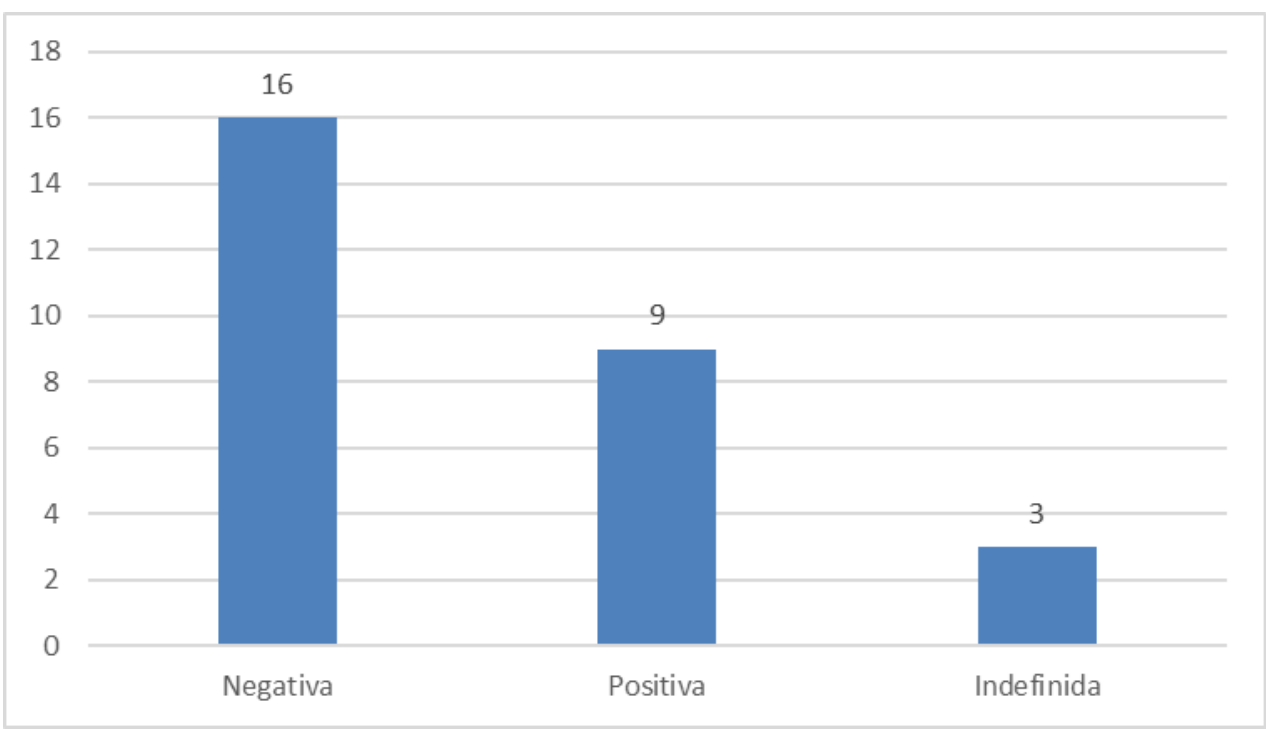

Fuente: elaboración propia en base a relevamientos realizados por los Observatorios de Medios de la Universidad Nacional de Quilmes, la Universidad Nacional de Cuyo y la Universidad Nacional del Centro de la Provincia de Buenos Aires. 
Gráfico 4: Frecuencia de cobertura de candidatos de Juntos por el cambio y del Frente de Todos según su valorización del tópico aborto.

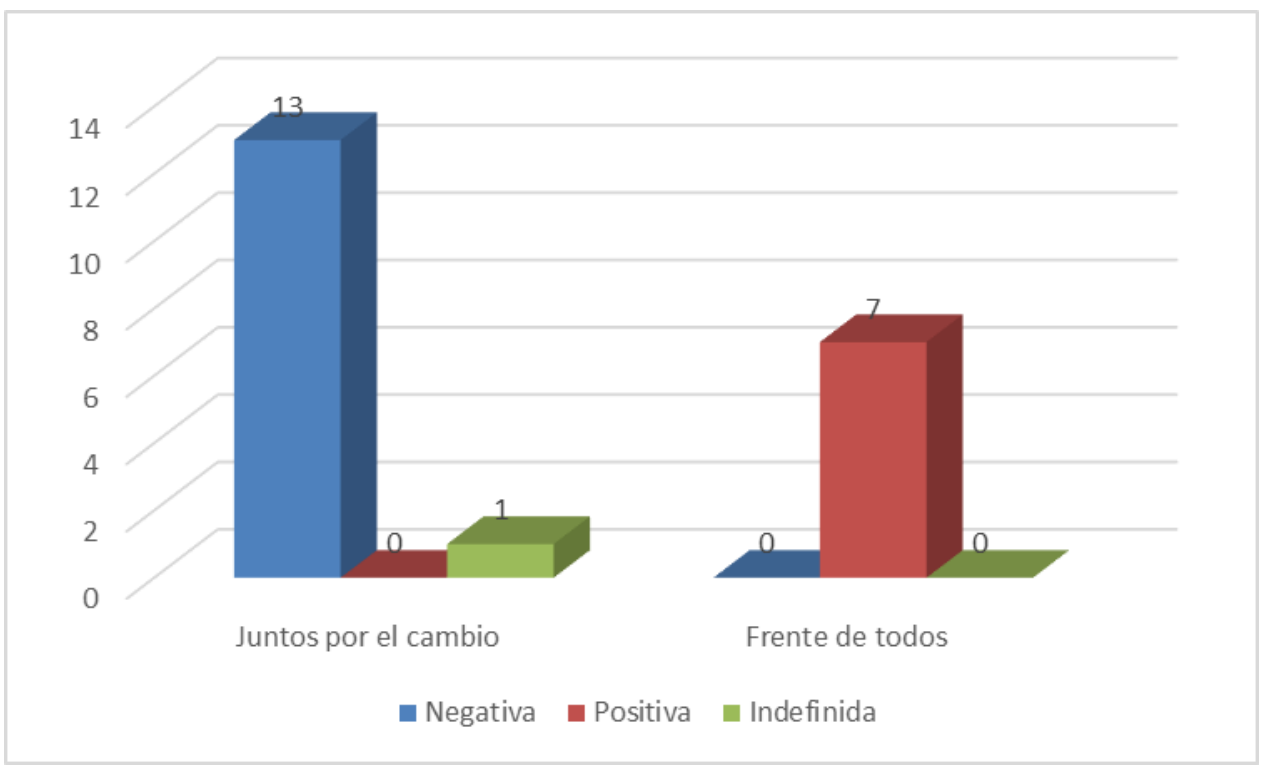

Fuente: elaboración propia en base a relevamientos realizados por los Observatorios de Medios de la Universidad Nacional de Quilmes, la Universidad Nacional de Cuyo y la Universidad Nacional del Centro de la Provincia de Buenos Aires

En los gráficos 3 y 4 se puede ver la predominancia de la valoración negativa sobre la legalización y/o despenalización del aborto. Esta valoración remite a la referencia a actores que han manifestado una postura contraria o bien en el texto de la noticia o en contextos ajenos a los narrados en la pieza informativa cuando se remite, por ejemplo, a "los celestes". En el gráfico 3 se computaron todas las manifestaciones a favor, en contra o indefinidas sobre la legalización y despenalización del aborto que están presentes en las 20 piezas informativas. En algunas de las piezas se hace referencia a más de una postura, motivo por el cual dichas frecuencias superan en cantidad a las notas analizadas.

En el gráfico 4 se cruza la valoración sobre el tópico con la adscripción política de los actores a los partidos que concentraron los votos a nivel nacional y en varias provincias y municipios 
del país7. La categoría Juntos por el Cambio reúne aquellas notas donde Mauricio Macri, María Eugenia Vidal y otros actores del oficialismo y del equipo de campaña del partido se manifiestan sobre el tópico aborto. Como se desprende del gráfico, con la sola excepción de una valoración indefinida, en 13 casos se encontraron valoraciones contrarias a la legalización del aborto por parte de estos actores. Por el contrario, aquellos actores pertenecientes al Frente de Todos (Alberto Fernández como candidato presidencial, y otras referencias a candidatos a nivel nacional y municipal) son vinculados en todos los casos con posturas favorables a la despenalización y legalización del aborto.

\section{Discusión}

La interpretación de la información que arrojan los gráficos será realizada a la luz de los resultados de las elecciones primarias del mes de agosto, que sorprendieron tanto al oficialismo como a la oposición por la distancia de más de 15 puntos entre el candidato presidencial del Frente de Todos, Alberto Fernández, y el candidato oficialista y presidente en ejercicio Mauricio Macri, de la coalición Juntos por el Cambio.

Como plantearon Page y Antenucci (2019): "El resultado de las PASO presidenciales del 11 de agosto tomó a todos por sorpresa. Supuestamente no se elegía nada, pero se redefinió todo" (p. 1). No sólo impactó la brecha entre los primeros dos candidatos, sino también el nivel de concentración de los votos entre esas dos opciones (Koziner, 2020; Leiras, 2019; Reina, 2019). En adelante, el oficialismo se vio obligado a repensar la campaña de cara a los comicios de octubre y doblegó esfuerzos en dos direcciones: profundizando la polarización con los candidatos del Frente de Todos e intentando cosechar votos que se habían volcado en primera vuelta por partidos de la oposición de la centroderecha y que les permitan alcanzar en octubre un porcentaje que los posicione en un escenario de ballotage.

De todas formas, y debido a la dificultad de reducir la brecha de 15 puntos en pocos meses y en el marco de la espiral de la crisis económica- se comenzó a posicionar a Alberto Fernández como el sucesor. En su oficina en Buenos Aires comenzaron a circular con mayor frecuencia

\footnotetext{
7 Es por ello que fueron excluidas del gráfico las valoraciones de aquellos actores que integran otras fuerzas políticas o sobre los cuales no se puede identificar pertenencia a un partido (como actores de la sociedad civil o sectores religiosos).
} 
diversos actores de los arcos político, empresarial, sindical y social. Su agenda incluyó, además, visitas al extranjero poco tiempo antes de los comicios de octubre.

Como destacaron varios autores (Garriga \& Negri, 2020; Koziner, 2020; Ratto, 2020; Reina, 2019) la crisis económica se impuso como tópico central en la campaña electoral de 2019, dejando poco margen para la discusión sobre otras cuestiones que también estaban en la agenda política y pública hasta hace un año atrás. En este sentido, se explica el porcentaje del $1 \%$ que ocuparon las coberturas que abordaron simultáneamente las elecciones y el debate sobre el aborto, contrariando las expectativas que se tenían en 2018 sobre el peso que mantendría la cuestión en la agenda política del año electoral.

Si bien la frecuencia de cobertura fue casi análoga entre ambos períodos sobre los cuales se realizó el relevamiento -9 piezas informativas en fechas próximas a las PASO y 11 en las fechas cercanas a las elecciones generales- la diferencia entre ambas instancias radicó en el lugar que tuvo el tópico en las noticias (Tablas 1 y 2). En el primer período no hubo noticias donde se registre el aborto como tópico principal. Ello cambia en el segundo período, debido principalmente a lo ocurrido en un acto proselitista, cuando Mauricio Macri explicitó por primera vez en el marco de la campaña su posición “a favor de las dos vidas” y en contra, por lo tanto, de la legalización del aborto. Esta actuación se leyó en varios portales como "una apuesta final y desesperada" (González, 2019) por captar votos de sectores religiosos y quitar los propios de partidos minoritarios que habían hecho de la oposición a la legalización del aborto uno de sus ejes de campaña ${ }^{8}$.

Lo trascendido de aquel acto marcó también un desplazamiento de la arena política al espacio público en lo referente a la aparición de Mauricio Macri en coberturas donde se aborda tanto la campaña electoral como el tópico de legalización del aborto. Antes de las PASO, el punto de encuentro entre ambos tópicos y la figura del presidente radicaba en el armado de las listas de candidatos al parlamento, donde se buscó alternar "verdes" y "celestes" e incorporar referentes de comunidades evangélicas (Bravo, 2019; Lejtman, 2019).

\footnotetext{
${ }^{8}$ Como el frente NOS, encabezado por Juan José Gómez Centurión y Cynthia Hotton.
} 
Del lado de la oposición del Frente de Todos, Fernández se pronunció como candidato a favor de la legalización del aborto recién hacia el final de la campaña y en el marco del debate con todos los candidatos. Anteriormente, su discurso establecía matices entre las prácticas de despenalizar y legalizar. Consideraba la primera como un paso previo necesario "para poner fin a la situación de clandestinidad", mientras que el horizonte de posibilidad de la legalización le resultaba más lejano y estaba sujeto a que se vuelva a "dar un debate que permita al Estado involucrarse" (Jastreblansky \& Sued, 2019).

Sin que pueda concluirse que el debate por el aborto fuera un tópico relevante en las coberturas informativas sobre la campaña, cabe destacar que 2019 representó un hito en lo que hace a su incorporación en la agenda de la campaña, por más que su tratamiento haya sido poco frecuente. Si contrastamos con la campaña electoral de 2015, las cuestiones de género no estaban siquiera presentes entre los tópicos de las coberturas relacionadas con las elecciones (Zunino \& Ortiz-Marín, 2017), a pesar de lo que significó socialmente la emergencia del movimiento Ni Una Menos aquel año. En cambio, en 2019 los temas de género sí estuvieron presentes en la agenda (Koziner, 2020), aunque marginalmente, e incluso fueron materia del debate televisado entre los candidatos presidenciales, quienes debieron expresarse en relación con el eje temático "Diversidad y género" en el primer debate realizado.

\section{Reflexiones finales}

El análisis del lugar que ocupó la legalización del aborto en la campaña electoral tuvo como meta aportar al conocimiento sobre la visibilización de asuntos y problemas públicos en las agendas políticas y mediáticas en circunstancias clave de las democracias. Se buscó reflexionar sobre el modo en que aparecen en las propuestas de los diferentes candidatos los temas que importan a la opinión pública en un momento dado.

2018 resultó un año bisagra para el movimiento feminista y se tuvo la expectativa de que un recambio de gobierno en 2019 no solo habilitaría la nueva presentación del proyecto de legalización del aborto sino también su efectiva sanción en un Congreso que renovaba una porción significativa de las bancas en las dos cámaras. Para ello, la inclusión del tema en la agenda de la campaña parecía ser vital. No obstante, el análisis realizado demostró que su presencia fue más bien marginal. 
Podríamos entonces abrir el interrogante si para los votantes era en efecto relevante para definir su voto la postura que los candidatos adoptaron acerca de la legalización del aborto, en vistas a la escasa visibilidad que el tema tuvo durante la campaña. Esta duda, cuya respuesta está fuera del alcance de los resultados de este trabajo, se basa en una de las premisas centrales de la teoría de la agenda setting, que remarca la coincidencia en las agendas mediática y pública en cuanto a la relevancia asignada a ciertos tópicos. En este trabajo, el aporte se centró en la medición de la relevancia del tópico aborto en las coberturas informativas sobre las elecciones en los medios digitales más consultados en el país.

La poca relevancia que obtuvo la temática en estos medios permitió focalizar el análisis de contenido sobre la totalidad de las piezas informativas que lo abordaban, analizando los actores que allí aparecían y la valorización que hacían sobre la cuestión. Los resultados permitieron concluir que prevalecieron las valoraciones negativas sobre la legalización del aborto en coberturas sobre las elecciones donde los actores de la noticia eran candidatos de Juntos por el Cambio.

En definitiva, si bien puede decirse que la agenda de género tuvo lugar en la campaña electoral, no primaron las coberturas relacionadas a la ampliación de derechos y la referencia al tópico legalización del aborto tuvo mayor presencia entre noticias que se relacionaban a estrategias direccionadas a captar votos de sectores conservadores en la materia. No obstante, para evaluar un mayor alcance de estas conclusiones, en futuras investigaciones se ampliarán las unidades de contexto para atender a posibles contrastes entre medios con líneas editoriales divergentes entre sí.

A pesar del lugar marginal que el tópico ocupó en la agenda política durante las elecciones, en el marco de la apertura de sesiones ordinarias en el Congreso en el año 2020, el flamante presidente Alberto Fernández anunció que el Ejecutivo enviaría un proyecto de legalización del aborto para ser debatido nuevamente. En un año sumamente atípico -signado por los efectos de la pandemia Covid-19 que trastocó todas las agendas- el proyecto fue finalmente enviado, nuevamente discutido, igualmente cuestionada la "urgencia" de su tratamiento por sectores que se oponían a su sanción (Altieri \& Rosenberg, 2020), y votado dos días antes de que termine el año. En contraste con lo ocurrido en 2018, el resultado no fue ajustado: 38 votos afirmativos, 29 negativos y 1 abstención. Este cambio significativo obedeció en parte a la reconfiguración del mapa político en el congreso tras las elecciones de 2019; será materia 
para otras investigaciones poder indagar en el modo en que la lucha feminista pudo participar en esa reconfiguración, así como en el orden de prioridades de la agenda política.

\section{Agradecimientos}

A la Red de Observatorios de Medios que integran la Universidad Nacional de Cuyo, La Universidad Nacional del Centro de la Provincia de Buenos Aires y la Universidad Nacional de Quilmes por brindarme acceso al relevamiento de las noticias del período electoral de 2019 en Argentina.

A todos los integrantes del Observatorio de la Universidad Nacional de Quilmes, por los conocimientos compartidos, gracias a los cuales pude elaborar este trabajo.

A Natalia Aruguete y a Nadia Koziner por su acompañamiento y orientación en la investigación realizada.

\section{Financiamiento}

Esta investigación fue posible gracias al financiamiento del Consejo Nacional de Investigaciones Científicas y Técnicas y del Observatorio de Medios de la Universidad Nacional de Quilmes.

\section{Conflicto de interés}

La autora declara que no existen conflictos de interés. Los datos obtenidos en este estudio fueron construidos a partir de un relevamiento de noticias realizado por la Red de Observatorios anteriormente mencionada, a la cual se desliga de cualquier error u omisión que pueda existir en el presente estudio. 


\section{Referencias bibliográficas}

ALCARAZ, M. F. (2019): iQue sea Ley! La lucha de los feminismos por el aborto legal. Buenos Aires, Marea editorial.

ALCONADA-MON, H. (1 de abril 2018): ¿Cuál es la apuesta de Macri con el aborto? The New York Times. https://www.nytimes.com/es/2018/04/o1/espanol/opinion/opinionalconada-macri-aborto-argentina.html

ALEXA (2020): Top Sites in Argentina. Alexa. http://www.alexa.com/topsites/countries/AR

ALTIERI, D. \& ROSENBERG, L. (30 de noviembre 2020): La consideración del proyecto como un intento por correr el foco sobre asuntos "más urgentes". El anuncio en Twitter, la repercusión y las agendas. Letra P. Medios y redes ante la intervención del Ejecutivo en el debate del aborto. www.letrap.com.ar/nota/2020-11-30-14-33-0-medios-y-redes-ante-laintervencion-del-ejecutivo-en-el-debate-del-aborto?fbclid=IwAR30yG-MhVbiuYRxCQBogOcAgpYhTIkJZXxU -DpjDRJ321GEcs Zg8gZU

AMADEO, B. (2018): Argentina 2019: de un ambiente de cambio a un cambio de ambiente. En A. Arellano (Coord.), Nuevas campañas electorales en América Latina (pp. 101-122). Montevideo, Konrad Adenauer Stiftung.

ARUGUETE, N. (2015): El poder de la agenda. Buenos Aires, Biblos.

BARRANCOS, D. (2018): La ley abortada: Notas sobre el debate de la interrupción voluntaria del embarazo. Salud Colectiva, 14(3), pp. 373.

https://doi.org/10.18294/sc.2018.2025

BECERRA, M. \& MASTRINI, G. (2017): La concentración infocomunicacional en América Latina (2000-2015). Buenos Aires, Universidad Nacional de Quilmes Editorial. 
BRAVO, M. (22 de julio 2019): Mauricio Macri busca el voto evangélico para compensar la distancia de la Iglesia Católica. Clarín. https://www.clarin.com/politica/mauricio-macribusca-voto-evangelico-compensar-distancia-iglesia-catolica o $\mathrm{CNe3e}$ Kcc.html

CARBAJAL, M. (9 de agosto 2018): Ganamos. Página/12.

https://www.pagina12.com.ar/134081-ganamos

ENTMAN, R. M. (2003): Cascading Activation: Contesting the White House's Frame After 9/11. Political Communication, 20(4), pp. 415-432.

DOI: http://doi.org/10.1080/10584600390244176.

FALCONE, M. (2019): El gobierno no será reelecto. POSTdata, 24(1), pp. 221-230.

GARRIGA, A. C. \& NEGRI, J. (2020): It's (Almost) Always the Economy: Economic Performance and Political Realignments in Argentina in 2019. Revista de Ciencia Política, 40(2), pp. 137-161. http://dx.doi.org/10.4067/So718-090X2020005000104

GONZÁLEZ, F. (9 de octubre 2019): La apuesta final y desesperada de Mauricio Macri. Clarín.

https://www.clarin.com/politica/apuesta-final-desesperada-mauricio-macri $\quad$ o 5 cF1eu8Q

INSTITUTO VERIFICADOR DE CIRCULACIONES (IVC) (2020): Boletín Xpress. IVC. https://www.ivc.org.ar/boletin-xpress/

JASTREBLANSKY, M. \& SUED, G. (28 de julio 2019): Macri y Alberto Fernández definieron sus propuestas. La Nación. https://www.lanacion.com.ar/politica/los-candidatosdefinieron-sus-propuestas-nid2271931

KOZINER, N. S. \& ARUGUETE, N. (2020): El conflicto mapuche en la prensa chilena. Anotaciones teórico-metodológicas para el análisis de los encuadres mediáticos. Perspectivas de la Comunicación, 13(1), pp. 203-217. 
KOZINER, N. S. (2013): Antecedentes y fundamentos de la teoría del framing en comunicación. Austral Comunicación, 2(1), pp. https://doi.org/10.26422/aucom.2013.0201.koz

KOZINER, N. S. (2020): Temas y fuentes en medios digitales argentinos. Un estudio en contexto electoral (2019). Más Poder Local, 40, pp. 46-56.

LEIRAS, M. (15 de agosto 2019): Mejor que decir es hacer cosas con palabras. Infobae. https://www.infobae.com/opinion/2019/08/15/mejor-que-decir-es-hacer-cosas-conpalabras/

LEJTMAN, R. (23 de julio 2019): Macri tiene el voto de la comunidad judía, seduce a los evangélicos y pelea por el apoyo católico. Infobae. https://www.infobae.com/politica/2019/07/23/macri-tiene-el-voto-de-la-comunidadjudia-seduce-a-los-evangelicos-y-pelea-por-el-apoyo-catolico/

MCCOMBS, M. \& SHAW, D. (1972): The agenda setting function of the mass media. Public Opinion Quarterly, 36, pp. 176-187.

MOM (2019): ¿Quiénes son los dueños de los medios en Argentina? Media Ownership Monitor Agentina.

https://argentina.mom-rsf.org/fileadmin/rogmom/output/argentina.momrsf.org/argentina.mom-rsf.org-es.pdf

PAGE, M. \& ANTENUCCI, P. (6 de noviembre 2019): Otra mirada de la elección presidencial 2019. OEAR. https://oear.cippec.org/novedades/otra-mirada-a-la-eleccion-presidencial2019/

PEKER, L. (2019): La revolución de las hijas. Barcelona, Paidós.

RATTO, M. C. (2020): Otra vez la economía. La influencia de la agenda económica en las elecciones 2019. Más Poder Local, 40, pp. 38-45. 
REINA, A. (2019): Polarización, crisis y debates presidenciales. Notas sobre la campaña electoral argentina del 2019. Más Poder Local, (39), pp. 34-36.

SÁDABA-GARRAZA, M. T. (2001): Origen, aplicación y límites de la teoría del encuadre (framing) en comunicación. Comunicación y Sociedad, XIV(2), pp. 143-175.

SVAMPA, M. (2019): Posprogresismos, polarización y democracia en Argentina y Brasil. Nueva Sociedad, 282, pp. 121-134.

TARDUCCI, M. (2018): Escenas claves de la lucha por el derecho al aborto en Argentina. Salud Colectiva, 14(3), pp. 425-432. https://doi.org/10.18294/sc.2018.2036

VOMMARO, G. (2018): ¿Cuán progre puede ser el macrismo? Revista Anfibia. http://revistaanfibia.com/ensayo/cuan-progre-puede-macrismo-2/

ZUNINO, E. \& ORTIZ-MARÍN, M. O. (2017): Los medios y las elecciones: la agenda informativa de la campaña presidencial de 2015 en la argentina. Más Poder Local, 30, pp. 56-66.

ZUNINO, E. (2016): La relevancia de las noticias en la prensa gráfica. Una reflexión teóricometodológica a partir del análisis del conflicto entre las corporaciones agrarias y el gobierno argentino. Comunicación y Sociedad, (25), pp. 127-156.

https://doi.org/10.32870/cys.voi25.4424 